\title{
Predicting Breakdown of the Continuum Equations Under Rarefied Flow Conditions
}

\author{
Iain D. Boyd \\ Department of Aerospace Engineering, University of Michigan, Ann Arbor, MI 48109
}

\begin{abstract}
The breakdown of the continuum equations of gas dynamics under conditions of rarefied flow is considered. The conditions of breakdown are of interest in assessing the physical accuracy of continuum solutions, in understanding the mathematical relations between different sets of continuum transport equations, and play a significant role in the development of hybrid methods that use both continuum and kinetic simulation techniques. A number of previous breakdown parameters are reviewed. A new method, based on kinetic theory analysis, is introduced for detecting conditions where the continuum approach is valid. Continuum breakdown is discussed in detail for gas expansions and shock waves by considering the basic physical phenomena involved in each case. Success of the breakdown parameters for these flows is assessed. It is shown that continuum breakdown in gas expansions and shock waves proceeds through different physical mechanisms that are best predicted by different types of breakdown parameters. The new method for detecting continuum onset is applied to shock waves and shown to offer promising results.
\end{abstract}

\section{INTRODUCTION}

The failure of the continuum equations of gas dynamics under certain physical conditions is the reason why there is a field of rarefied gas dynamics. In general, it is widely recognized that a continuum approach is invalid when the Knudsen number is large and this occurs for flows with low density and/or small characteristic length scales. In a global sense, a large Knudsen number means that there is not enough physical space to allow the gas to undergo a sufficient number of collisions to maintain equilibrium velocity distribution functions. At sufficiently large global Knudsen numbers, an entire gas flow system must be modeled using non-continuum, kinetic methods. However, in many gas flows of interest, the global Knudsen number may be small and yet there are localized regions where the continuum approach may fail. This article is motivated by the need for prediction of the failure of the continuum equations in these types of flows with a mixture of continuum and kinetic flow regions.

The most fundamental model of dilute gas dynamics is the Boltzmann equation that describes the evolution of the velocity distribution function of molecules. By taking moments of the Boltzmann equation, Maxwell derived his equation of change [1]:

$$
\frac{\partial(n\langle W\rangle)}{\partial t}+\frac{\partial\left(n\left\langle u_{i} W\right\rangle\right)}{\partial x_{i}}=\Delta[W]
$$

in which $W(\mathbf{C})$ is some molecular property that depends on the random velocity $\mathbf{C}=\left(\mathrm{C}_{\mathrm{i}}, \mathrm{C}_{\mathrm{j}}, \mathrm{C}_{\mathrm{k}}\right)$, $<>$ indicates an average over the velocity distribution function, and $\Delta[]$ indicates the change due to collisions. For example, if $W$ is the mass of a molecule, then in the absence of chemical reactions the mass of the particle is conserved in a collision, and Eq. (1) becomes the well-known continuity equation of continuum gas dynamics. In addition, if equilibrium is assumed, and $W$ is set to the momentum vector and the energy of a molecule, Eq. (1) provides a set of five continuum transport equations that is often called the Euler equations. The equation set corresponds to the case where the velocity distribution function everywhere in the flow is of the equilibrium Maxwellian form. To derive higher order sets of transport equations from Maxwell's equation of change, it must be noted from Eq. (1) that the temporal derivative of any moment $W$ depends on the divergence of the next higher velocity moment. This problem is addressed by one of two methods. In the Chapman-Enskog approach, a specific form of the velocity distribution function is assumed for flows perturbed slightly from the equilibrium state. In Grad's method of moments, specific relations are assumed between the second and fourth order velocity moments. Setting $W=m C_{i} C_{j}$ and $W=m C_{i} C_{j} C_{k}$ in 
Eq. (1), using either the Chapman-Enskog or the Grad methods, leads to a set of twenty equations (the 20-moment equations) consisting of the five Euler equations, five further equations involving the shear stress tensor $\tau_{i j}$, and ten further equations involving the symmetric heat flux vector $\mathrm{Q}_{\mathrm{ijk}}$. For modeling viscous continuum flow, a set of transport equations called the Navier-Stokes (NS) equations is regularly employed. The NS equations are obtained from the 20 -moment equations by replacing the heat flux tensor with a heat flux vector. As will be discussed later, this connection between the 20-moment equations and the NS equations may offer a convenient approach for detecting the onset of continuum conditions from within a kinetic simulation method.

In addition to the mathematical interest in the relations between different sets of transport equations, continuum breakdown is an important aspect of the development of hybrid computational techniques that combine the advantages of continuum and kinetic methods. The NS equations are solved with numerical efficiency by a variety of Computational Fluid Dynamics (CFD) techniques. For this reason, it is desirable to use these equations wherever possible within a given flow field. Particle-based kinetic methods such as the direct simulation Monte Carlo method (DSMC) [2] are physically accurate for nonequilibrium flow conditions, but are in general more numerically expensive than most CFD techniques. Therefore, the development of a hybrid DSMC-CFD method has the potential to provide physically accurate and numerically efficient solutions to flow problems involving regions of continuum and kinetic flow regimes. Examples of such flows may be found in hypersonic aerodynamics [3], micro-scale gas flows [4], and jet expansions [5] among others. A variety of hybrid methods have been proposed in previous investigations. There exist weakly coupled schemes $[5,6]$ for which a continuum solver provides a boundary condition for a particle method. There are also overlapping coupling strategies for which a particle method provides a boundary condition for a continuum scheme [7], or even provides transport coefficients for a continuum method [8]. Wadsworth and Erwin simulated one-dimensional shock waves [9] and two-dimensional slit flows [10] using a property extrapolation technique. Roveda et al. [11] described a Euler/particle approach that can analyze unsteady flows by coupling an adaptive discrete velocity (ADV) Euler solver and the DSMC method with an adaptive interface. In a following paper [12], they successfully simulated an unsteady pressure driven slit flow with the scheme. There are other adaptive hybrid schemes, such as the adaptive domain decomposition method proposed by Tiwari and Klar [13], and the adaptive mesh and algorithm refinement (AMAR) method developed by Garcia et al [14]. Recently, hybrid techniques based on a combination of the NS equations and the DSMC-IP method [15] were proposed for micro-scale flows [4] and hypersonic flows [16]. A critical aspect of any hybrid method involves the prediction of when to switch between the continuum and kinetic simulation methods. This is usually performed using continuum breakdown parameters that are evaluated from the continuum solution to identify regions of a flow field that require the more detailed kinetic treatment. An important motivation for the study reported here was a difficulty encountered in predicting continuum breakdown in a hybrid method for hypersonic flows [3].

The layout of the paper is as follows: first, the physical nature of continuum breakdown is discussed for gas expansions and for shock waves. Then, a number of different, existing breakdown parameters are discussed. It is shown that most of these can be linked to the Chapman-Enskog distribution function. A new parameter for detecting the onset of continuum flow within a kinetic method is proposed. Several of the breakdown parameters are then assessed using these simple flows. For shock waves, the new continuum onset parameter is investigated and found to provide promising results.

\section{PHYSICAL PROCESSES OF CONTINUUM BREAKDOWN}

\section{Gas Expansion}

As a gas expands rapidly from an orifice, the temperature and density decrease along the jet axis and thus to a decrease in the molecular collision rate. This behavior first leads to freezing of the internal energy modes in which the temperatures of the vibrational and rotational modes eventually level out to values higher than the translational temperature. Such behavior can be simulated using both continuum and kinetic methods. The translational temperature itself can be considered in terms of one mode in the direction parallel to the jet axis, and two modes perpendicular to the jet axis. At large distances from the jet source, the population of molecules close to the axis must have very small thermal velocities in the perpendicular direction, otherwise they would drift away from the axis. Therefore, in the absence of collisions, the translational temperature of the perpendicular modes continues to decrease with distance away from the source. However, the parallel translational mode does not experience this geometric effect and it's temperature will only continue to decrease while there are collisions to provide mixing 
between the perpendicular and parallel modes. Therefore, the parallel translational energy mode also freezes at a temperature that is higher than that corresponding to the perpendicular modes. These phenomena have been measured experimentally by Cattolica et al. [17] and computed using DSMC by Bird [18] in free jet expansions.

\section{Shock Waves}

Very different physical processes govern the breakdown of the continuum approach in shock waves. In the frame of a stationary wave, a shock wave involves the mixing of two equilibrium populations of gas that have very different temperatures and velocities. The mixing of the velocity distribution functions of these two populations occurs over a very small length scale of a few mean free paths through the mechanism of molecular collisions. Nonequilibrium velocity distribution functions in the middle of a hypersonic shock wave were measured experimentally and computed using DSMC by Pham-Van-Diep et al. [19].

\section{BREAKDOWN PARAMETERS}

\section{The Chapman-Enskog Parameter}

As discussed above, one of the methods for deriving transport equation sets from Maxwell's equation of change is the Chapman-Enskog approach in which the following form for the velocity distribution function is assumed:

$$
\begin{gathered}
f(\vec{C})=f_{0}(\vec{C}) \Gamma(\vec{C}), \quad \vec{C}=\vec{c} /(2 k T / m)^{1 / 2} \\
f_{0}(\vec{C})=\frac{1}{\partial^{3 / 2}} e^{-C^{2}} \\
\Gamma(\vec{C})=1+q_{i}^{*} C_{i}\left(\frac{2}{5} C^{2}-1\right)-\tau_{i j}^{*} C_{i} C_{j} \\
q_{i}^{*}=-\frac{\kappa}{P}\left(\frac{2 m}{k T}\right)^{1 / 2} \frac{\partial T}{\partial x_{i}}, \tau_{i j}^{*}=\frac{\mu}{P}\left(\frac{\partial v_{i}}{\partial x_{j}}+\frac{\partial v_{j}}{\partial x_{i}}-\frac{2}{3} \frac{\partial v_{k}}{\partial x_{k}} \delta_{i j}\right)
\end{gathered}
$$

where $f_{o}$ is the equilibrium Maxwellian form of the distribution. The Chapman-Enskog parameter, $\Gamma$, is a good predictor of non-continuum conditions. When $\Gamma$ is slightly perturbed from unity, then the 20 -moment (or perhaps the Navier-Stokes) equations are valid. When $\Gamma$ is sufficiently far from unity then these equation sets can be expected to fail, and a kinetic approach is required. The evaluation of $\Gamma$ is difficult because it involves computation of a heat flux $\mathrm{q}_{\mathrm{i}}{ }^{*}$ and a shear stress $\tau_{\mathrm{ij}}{ }^{*}$ that each involve flow field gradients. In addition, $\Gamma$ is a function of the random velocity $\mathrm{C}$ and so either an average or maximum value of $\Gamma$ must be evaluated over the full distribution function. This difficulty has motivated the development of a number of different, simpler breakdown parameters that tend to involve flow gradients, and/or shear stress and heat flux terms. Some of these parameters are reviewed in the following.

\section{Bird's Parameter for Expanding Flows}

Following on from the discussion above on the physical processes leading to continuum breakdown in gas expansions, it was recognized by Bird [18] that these phenomena are best considered in the frame moving with the flow. This led Bird [18] to propose the following criterion for continuum breakdown in expanding flows:

$$
P=-\frac{1}{v}\left(\frac{D(\ln \rho)}{D t}\right)=-\frac{\sqrt{\pi}}{2} s \frac{\lambda}{\rho} \frac{d \rho}{d x}>0.04
$$

where $v$ is the collision rate, $\rho$ is the density, $D$ is the substantive derivative, $s$ is the speed ratio, and $\lambda$ is the mean free path. Note that the second expression involving just the spatial derivative is for steady flows. Bird derived this criterion from DSMC computations and the usefulness of the parameter was illustrated by Cattolica et al. [17] through direct comparisons with experimental measurements of the parallel and perpendicular temperatures in a free jet expansion. 


\section{Gradient Length Local Knudsen Number}

An evaluation of different breakdown parameters was performed for hypersonic flows over a blunt body by Boyd et al. [20] involving the direct comparison of DSMC and NS solutions. It was discovered that Bird's parameter was unable to predict continuum breakdown in the region next to the body surface. This failure arises from the fact that the flow velocity approaches zero in this region giving very small values of $P$. An alternative parameter was proposed entitled the gradient-length-local Knudsen number, in which a local Knudsen number is evaluated using a length scale determined by the gradient of different flow properties $Q$ :

$$
K n_{G L L-Q}=\frac{\lambda}{Q}\left|\frac{d Q}{d x}\right|>0.05
$$

This criterion was found to successfully predict continuum breakdown in the shock wave and at the body surface in hypersonic blunt body flows. The same parameter has been evaluated in recent studies of hypersonic interacting flows by Wang and Boyd [3]. The maximum of the local Knudsen numbers based on density, temperature, and velocity was found to correctly predict continuum breakdown at the body surface and at the downstream edge of the shock wave, but was unsuccessful in predicting continuum breakdown in the shock front. We will return to this difficulty later. Note that the following relation exists between Bird's parameter and the gradient-length-local Knudsen number based on density: $P \sim M a . K n_{G L L-\rho}$ where $M a$ is the Mach number. This indicates that the two parameters should exhibit similar behavior for Mach numbers of order unity.

\section{Simplified Chapman-Enskog Parameter}

As discussed above, evaluation of the Chapman-Enskog parameter $\Gamma$ for continuum breakdown is numerically expensive. This has motivated the suggestion of related, but simpler breakdown parameters. For example, Garcia et al. [14] proposed the following criterion:

$$
B=\max \left(\left|q_{i}^{*}\right|,\left|\tau_{i j}^{*}\right|\right)>0.2
$$

where the normalized heat flux and shear stress have the same definitions as given above for the Chapman-Enskog distribution function.

\section{A New Parameter for Detecting Continuum Onset}

As discussed in the introduction, the Navier-Stokes equations, that form the basis of most CFD approaches for viscous flows, may be derived from the 20 -moment equations by assuming the following relation between the full, symmetric heat flux tensor $\mathrm{Q}_{\mathrm{ijk}}$ and a heat flux vector, $\mathrm{h}_{\mathrm{i}}[1]$ :

$$
Q_{i, j, k}=\frac{2}{5}\left(\delta_{i, j} h_{k}+\delta_{i, k} h_{j}+\delta_{j, k} h_{i}\right)
$$

The following relations can then be deduced for one-dimensional flow in which the Navier-Stokes equations are valid:

$$
\frac{5}{6} Q_{1,1,1}=\frac{5}{2} Q_{1,2,2}=\frac{5}{2} Q_{1,3,3}=h_{1} ; Q_{1,1,2}=Q_{2,2,2}=Q_{3,3,2}=0
$$

These relations suggest a pathway for evaluating the approach of continuum onset within a particle simulation method such as DSMC. Specifically, the DSMC method can be used to evaluate directly elements of the heat flux tensor as follows:

$$
Q_{i, j, k}(D S M C)=m n \iiint c_{i} c_{j} c_{k} f\left(c_{i}, c_{j}, c_{k}\right) d c_{i} d c_{j} d c_{k}
$$

These elements can then be compared to see if they obey the relations in Eq. (10), and in this way provide a measure of the approach to continuum (NS) flow conditions within the DSMC computation. As will be shown in the discussions below, the ability to detect continuum onset within DSMC may be an important aspect of the development of hybrid DSMC-CFD methods for flows involving shock waves. 


\section{DISCUSSION}

In the following sections, results are provided to illustrate the nature of nonequilibrium and continuum breakdown in gas expansions and shock waves. In the case of shock waves, new results are presented to show the performance of the continuum onset parameter described above.

\section{Gas Expansion}

Figures $1 \mathrm{a}$ and $1 \mathrm{~b}$, taken directly from Cattolica et al. [17], illustrate the translational nonequilibrium processes described above for free jet expansions. As shown in Fig. 1a, due to the reduced collision rate at large distances from the jet source, the translational temperature mode parallel to the jet freezes while the perpendicular temperature modes continue to decrease due to the geometric effect. Figure $1 \mathrm{~b}$ demonstrates that Bird's criterion $P>0.04$ successfully predicts this type of continuum breakdown.
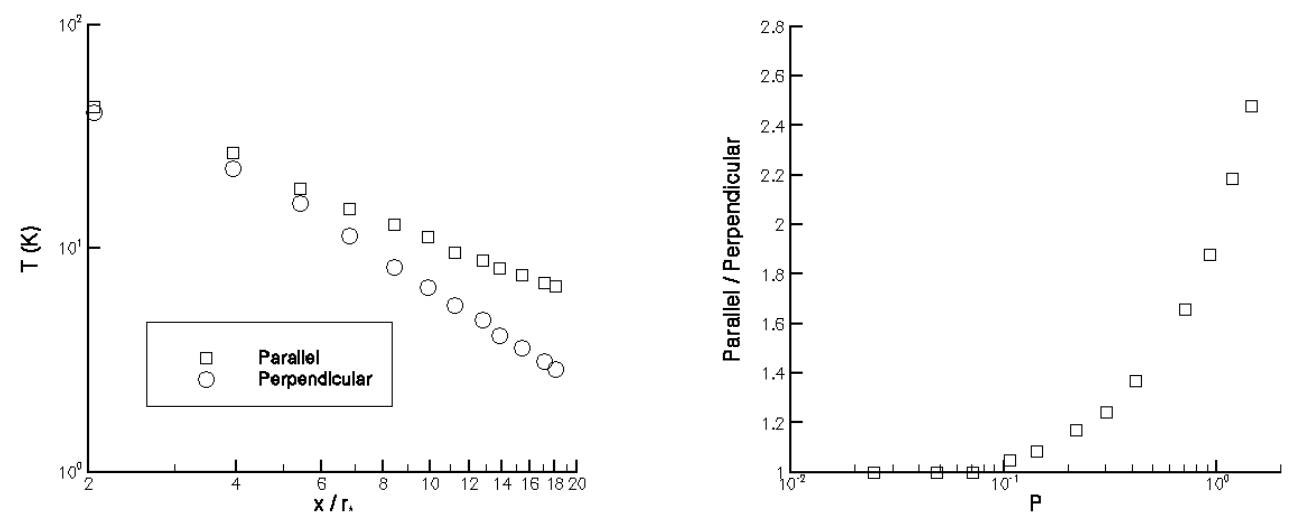

FIGURE 1. Translational nonequilibrium in freejet expansions (all data taken from Cattolica et al. [17]).

Evaluation of several continuum breakdown parameters was performed by George and Boyd [5] for expanding flows. Figures $2 \mathrm{a}$ and $2 \mathrm{~b}$ show results of experiments and computations for expansion of carbon dioxide through a supersonic nozzle. The measured data are from Bailey [21]. The comparisons between CFD solutions of the Navier-Stokes equations and the experimental data in Fig. 2a indicate failure of the CFD approach at a normalized radius of about 0.75 . Computation of the breakdown parameters $P, \mathrm{Kn}_{\mathrm{GLL}-\rho}$, and $\Gamma$ are shown in Fig. $2 \mathrm{~b}$. These indicate that any of the parameters considered can successfully predict continuum breakdown in this case.
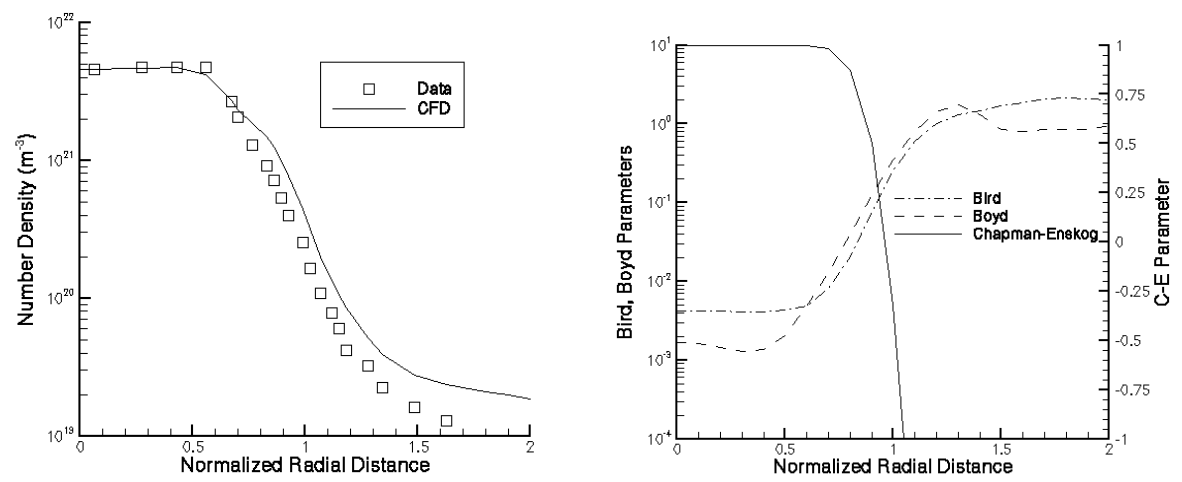

FIGURE 2. Continuum breakdown in a nozzle/plume flow of carbon dioxide (all data taken from George and Boyd [5]).

\section{Shock Waves}

Experimental measurements of velocity distributions function, e.g. see Pham-Van-Diep et al. [19], show that shock waves involve the collisional mixing over a very short distance (a few mean free paths) of two equilibrium 
populations of molecules that have very different temperatures and velocities. Within the structure of the shock wave, the velocity distribution function is highly nonequilibrium and continuum methods can be expected to fail. An illustration of this failure is shown in Fig. 3 where the reciprocal shock thickness (a measure of the density gradient at the center of the shock) is shown as a function of Mach number in argon gas. The experimental data are from Alsmeyer [22]. Clearly, the NS equations predict a significantly higher reciprocal thickness than the DSMC results and the measured data. Thus, the NS equations predict shocks that are much too thin.

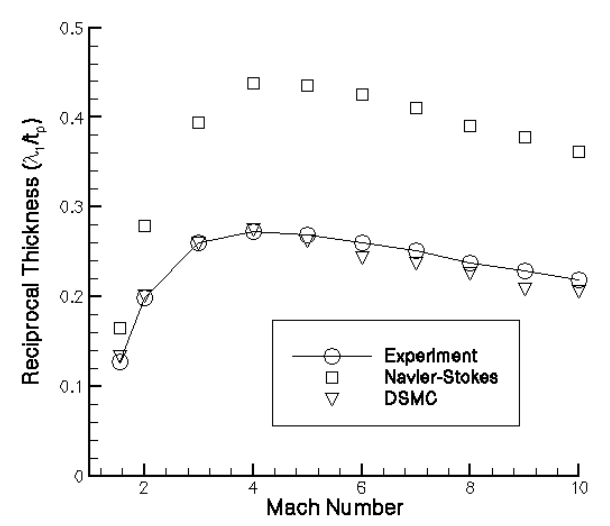

FIGURE 3. Reciprocal shock thickness for shock waves in argon.

Due to the strong degree of nonequilibrium in the shock, the Chapman-Enskog parameter $\Gamma$ should be able to predict continuum breakdown. Presumably due to its complexity, however, $\Gamma$ has never been applied to shock waves. Both the local Knudsen number, $K n_{G L L}$, and Garcia's parameter $B$ were introduced specifically for flows with shock waves. In recent work by Wang et al. [16] on development of a hybrid DSMC-CFD method for hypersonic flows, the $K n_{G L L}$ parameter was employed as the switching parameter between CFD and DSMC. The parameter was successful at predicting breakdown towards the downstream edge of the shock wave, and at the body surface. However, the parameter was not able to predict the onset of nonequilibrium at the upstream edge of the shock. This aspect is considered in greater detail here. In Figs. $4 \mathrm{a}$ and $4 \mathrm{~b}$, results are shown for a weak Mach 1.55 shock in argon. The DSMC and CFD profiles in Fig. 4a are in reasonably good agreement although the DSMC method predicts a slightly thicker shock. This is a case for which the NS equations are arguably still valid. Evaluation of various breakdown parameters shown in Fig. $4 \mathrm{~b}$ indicates that the breakdown thresholds for $\mathrm{Kn}_{\mathrm{T}}$ and $q_{i}$ are exceeded by a small amount confirming the conclusion that this is a flow at the edge of the continuum regime. Direct evaluation using DSMC of various elements of the heat flux tensor are shown in Figs. 5a and 5b. These show the trends expected for near-continuum conditions, i.e. they follow Eq. (10).
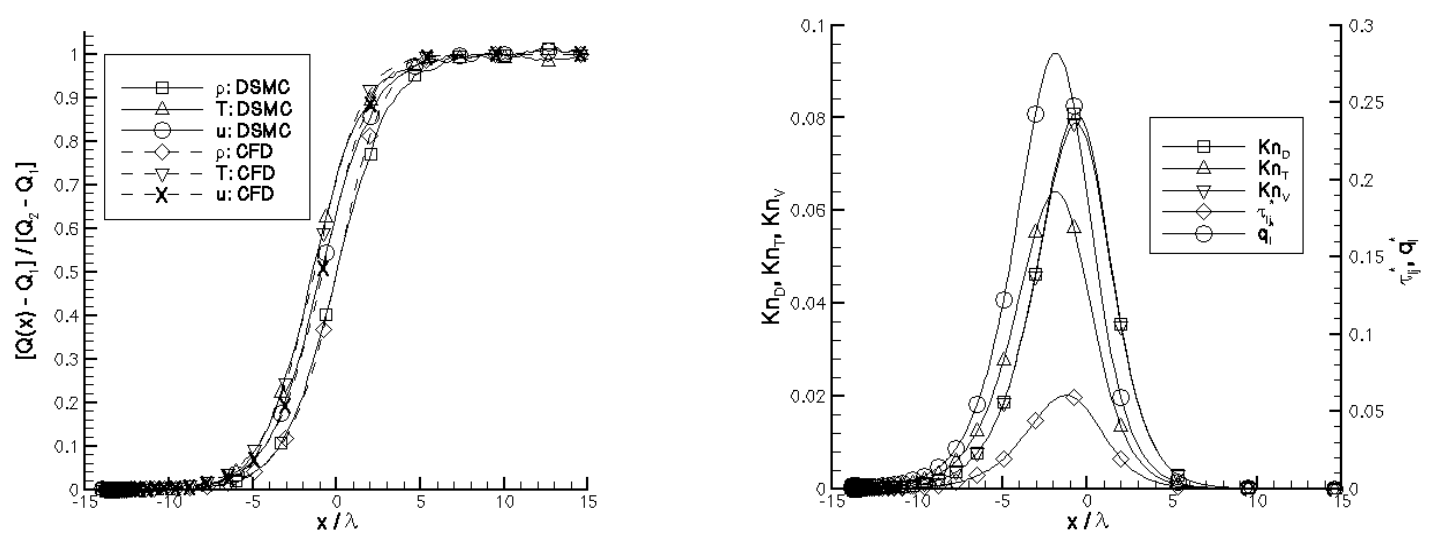

FIGURE 4. Profiles for a Mach 1.55 shock in argon: (a) DSMC and CFD flow properties; (b) breakdown parameters. 

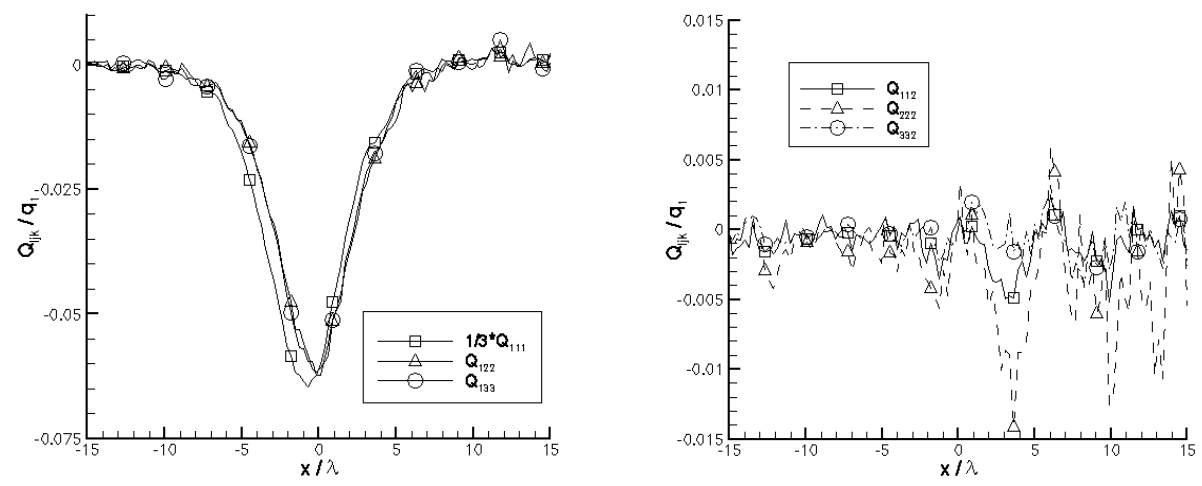

FIGURE 5. Profiles of heat flux tensor elements evaluated using DSMC for a Mach 1.55 shock in argon.
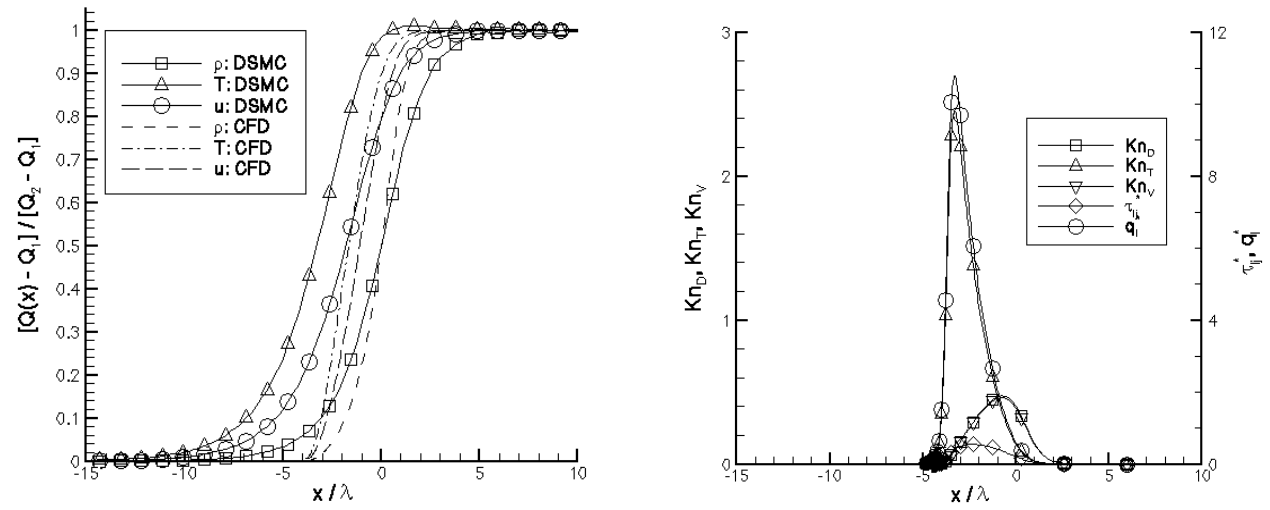

FIGURE 6. Profiles for a Mach 10 shock in argon: (a) DSMC and CFD flow properties; (b) breakdown parameters.
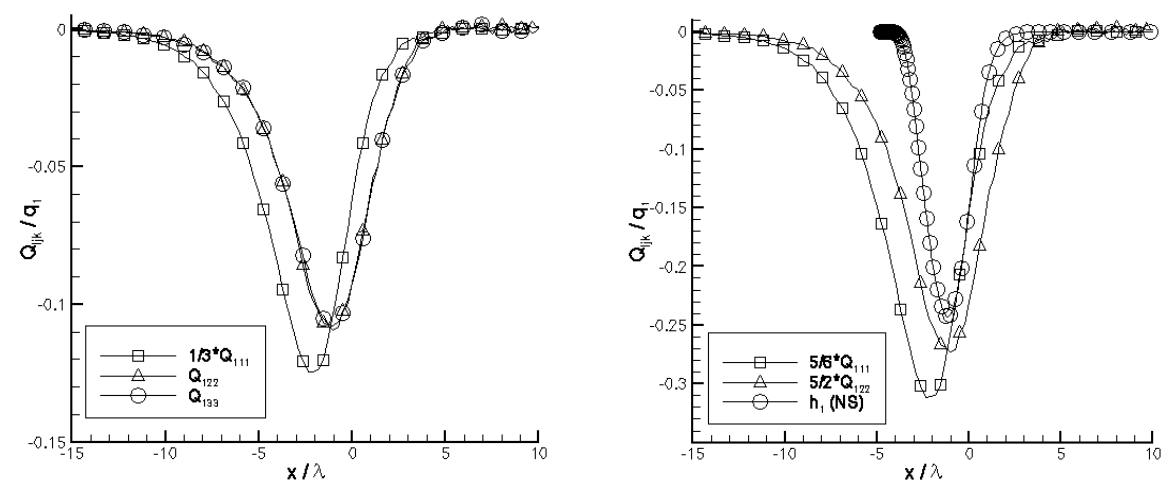

FIGURE 7. Heat flux profiles for a Mach 10 shock in argon: (a) DSMC evaluated terms of the tensor; (b) comparison of DSMC evaluated tensor and NS evaluated vector terms.

Now, a stronger shock wave with a Mach number of 10 in argon is considered. This is a case that should involve a higher degree of nonequilibrium. In Fig. 6a, a comparison of flow properties computed using CFD and DSMC shows significant differences. The shock thickness predicted by CFD is much smaller than that obtained with DSMC. Evaluation of the various breakdown parameters, as shown in Fig. 6b, indicates a clear case of continuum breakdown. However, these results illustrate the problem encountered in Ref. 16. That is, because the NS equations do not predict any change in properties before 5 mean free paths upstream of the shock center, there is no prediction of continuum breakdown there. In a hybrid DSMC-CFD method, switching from CFD to DSMC at the point indicated in Fig. $6 \mathrm{~b}$ is unlikely to lead to accurate simulation of the shock front, and this was indeed a finding of Ref. 
16. In Figs. 7, the heat flux properties of the Mach 10 argon shock are considered. In Fig. 7a, heat flux tensor terms evaluated using DSMC indicate nonequilibrium behavior, i.e. Eq. (10) is not obeyed. The heat flux element $\mathrm{Q}_{111}$ is not exactly three times larger than $\mathrm{Q}_{122}$ or $\mathrm{Q}_{133}$ and the location of initial disagreement overlaps with the point where the DSMC profiles first begin to rise in Fig. 6a. A further illustration of the usefulness of $\mathrm{Q}_{\mathrm{ijk}}$ in predicting continuum breakdown is shown in Fig. 7b where the DSMC evaluated tensor terms are compared with the NS evaluated heat flux vector. The lack of agreement between the DSMC and NS profiles again indicates breakdown.

\section{CONCLUDING REMARKS}

The nature of continuum breakdown in gas expansions and shock waves was shown to occur through different physical mechanisms. For gas expansion, the translational temperature in the direction of expansion freezes at a high value due to lack of collisions and separates from the translational temperatures in the direction perpendicular to the flow that continue to decrease due to a geometric effect. In a shock wave, two very different populations of molecules are mixed through collisions over the space of a few mean free paths leading to nonequilibrium velocity distribution functions. Prediction of these forms of continuum breakdown was shown to require different types of parameters. Various breakdown parameters have been proposed in the literature, and these can be traced to terms in the Chapman-Enskog distribution function and are based on flow field gradients, and the shear stress and heat flux. The existing parameters were unable to predict breakdown in the shock front because the NS equations modeled shocks that are too thin. A new continuum onset parameter involving direct evaluation of heat flux tensor elements was proposed to remedy this problem, and was found to offer promising results.

\section{ACKNOWLEDGMENTS}

The author gratefully acknowledges the support for this work from the Air Force Office of Scientific Research through MURI grant F49620-98-1-0433 with Dr. Arje Nachman as technical monitor, and again from the Air Force Office of Scientific Research through grant F49620-01-1-0003 with Dr. John Schmisseur as technical monitor.

\section{REFERENCES}

1. Gombosi, T.I., Gaskinetic Theory, Cambridge University Press, Cambridge, 1994.

2. Bird, G.A., Molecular Gas Dynamics and the Direct Simulation of Gas Flows, Oxford University Press, New York, 1994.

3. Wang, W.L. and Boyd, I.D., AIAA Paper 2002-0651 (2002).

4. Sun, Q., and Boyd, I.D., Journal of Computational Physics, 179, 400-425 (2002).

5. George, J., and Boyd, I.D., AIAA Paper 99-3454 (1999).

6. Hash, D.B., and Hassan, H.A., AIAA Paper 96-0353 (1996).

7. Bourgat, J.F., Tallec, P.L., and Tidriri, M.D., Journal of Computational Physics, 127, 227-245 (1996).

8. Oh, C.K., and Oran, E.S., AIAA Paper 98-0849 (1998).

9. Wadsworth, D.C., and Erwin, D.A., AIAA Paper 90-1690 (1990).

10. Wadsworth, D.C., and Erwin, D.A., AIAA Paper 92-2975 (1992).

11. Roveda, R., Goldstein, D.B., and Varghese, P.L., Journal of Spacecraft and Rockets, 35(4), 258-265 (1998).

12. Roveda, R., Goldstein, D.B., and Varghese, P.L., Journal of Spacecraft and Rockets, 37(6), 753-760 (2000).

13. Tiwari, S., and Klar, A., Journal of Computational and Applied Mathematics, 90, 223-237 (1998).

14. Garcia, A.L., Bell, J.B., Crutchfield, W.Y., and Alder, B.J., Journal of Computational Physics, 154, 134-155 (1999).

15. Fan, J., and Shen, C., Journal of Computational Physics, 167, 393-412 (2001).

16. Wang, W.-L., Sun, Q., and Boyd, I.D., AIAA Paper 2002-3099 (2002).

17. Cattolica, R., Robben, F., Talbot, L., and Willis, D.R., Physics of Fluids, 17(10), 1793-1807 (1974).

18. Bird, G.A., AIAA Journal, 8(11), 1998-2003 (1970).

19. Pham-Van-Diep, G., Erwin, D., and Muntz, E.P., Science, 245(4918), 624-626 (1989).

20. Boyd, I.D., Chen, G., and Candler, G.V., Physics of Fluids, 7(1), 210-219 (1995).

21. Bailey, A.B. and Price, L.L., AEDC-TR-85-26, (1985).

22. Alsmeyer, H., Journal of Fluid Mechanics, 74(3), 497-513 (1976). 